\title{
Thoracic Spinal Cord Ependymal Tumor
}

National Cancer Institute

\section{Source}

National Cancer Institute. Thoracic Spinal Cord Ependymal Tumor. NCI Thesaurus. Code C131603.

An ependymal tumor arising from the thoracic region of spinal cord. 